\title{
Radiodermatitis as a consequence of radiation recall induced by acyclovir: case report
}

\author{
Marcos Tumitan Zorzan ${ }^{1}$, Renata de Mello Pereira ${ }^{1}$, Lucas Farina Lima ${ }^{1}$, Tatiana Veri de Arruda Mattos ${ }^{2}$, \\ Rafael Sá ${ }^{1,3}$ \\ ${ }^{1}$ University of Western São Paulo, Presidente Prudente, Brazil \\ ${ }^{2}$ Santa Casa de São Paulo, São Paulo, Brazil \\ ${ }^{3}$ Federal University of São Paulo, São Paulo, Brazil
}

\begin{abstract}
Background: Radiation recall dermatitis (RRD) is an inflammatory reaction in an area of the skin previously irradiated for cancer treatment. The reaction usually occurs following the administration of a cytotoxic drug. Manifestations range from mild to severe, resulting in tissue necrosis. It is treated with removal of the probable causative agent, daily dressings and surgical debridement of the necrotic area.

Case presentation: A 54-year-old woman had a previous diagnosis of intraductal carcinoma in situ, and had been submitted to lumpectomy and adjuvant radiotherapy and hormonal therapy. One year after surgery, sores suggestive of herpes zoster infection developed, and treatment with acyclovir was started. At the same time, there was the onset of pain and fever. In the skin area previously irradiated, there was breast hardening, skin infiltration and serosanguinolent discharge. An incisional biopsy was performed to rule out radioinduced sarcoma. The patient was treated with surgical debridement.

Conclusions: This case report describes acyclovir as a possible trigger of RRD, a rare condition that could have been mistaken for an eruption with other causes. In this case, the dermatitis reaction was confined to the previously irradiated area of the skin, which suggested radiation recall. A better understanding of the condition's mechanism and about the possible joint effects of drugs and radiotherapy on the skin is necessary.
\end{abstract}

Key words: radiation recall dermatitis; radiodermatitis; radiotherapy; acyclovir; intraductal carcinoma in situ

Rep Pract Oncol Radiother 2021;26(3):475-480

\section{Introduction}

Radiation recall is defined as an inflammatory reaction on a region of the body previously irradiated by radiotherapy [1-3] usually occurring after administration of certain trigger agents, such as antineoplastic and chemotherapy drugs, antituberculosis medication, antibiotics, tamoxifen, simvastatin, and exposure to ultraviolet light [2]. The site most commonly affected by radiation recall is the skin, and in this case, it is described as radiation recall dermatitis (RRD) [3].

The manifestations of RDD vary in intensity and include maculopapular eruptions with erythema and vesicle formation, peeling of the affected skin and even severe skin necrosis, occurring days to years after the radiation, even when there was little or no residual reaction resulting from prior radiation sessions [1]. RRD is not necessarily an acute skin reaction during or at the end of the radiotherapy sessions [3].

Address for correspondence: Marcos Tumitan Zorzan, University of Western São Paulo, Presidente Prudente, Brazil, 19.050-920, tel: 0800-7715533; e-mail: marcostzorzan@gmail.com

This article is available in open access under Creative Common Attribution-Non-Commercial-No Derivatives 4.0 International (CC BY-NC-ND 4.0) license, allowing to download articles and share them with others as long as they credit the authors and the publisher, but without permission to change them in any way or use them commercially 
Radiotherapy uses high-energy or gamma rays directly on the tumor or on the affected site after the surgical intervention, promoting local control of the tumor and the death of cancer cells that remained after surgery $[4,5]$. Radiotherapy typically causes sensitivity up to 7 days after the sessions. $\mathrm{RRD}$, instead, is triggered after the use of drugs [6].

RRD is a well-known but poorly understood phenomenon, mainly because of the lack of animal models. There is a myriad of hypotheses that explain the pathophysiological of RDD, like vascular damage, epithelial stem cell inadequacy, epithelial stem cell sensitivity and drug hypersensitivity reaction [7]. The theory of alteration in vascular permeability argues that previous radiotherapy changes the pharmacokinetics of the inducing drug, promoting the inflammatory reaction [6]. The epithelial stem cell inadequacy hypothesis proposes that the radiation reduces the number of stem cell and overall skin stability, yielding a cell proliferation response triggered by the drug $[8,9]$. The epithelial stem cell sensitivity hypothesis relies on the fact that radiation promotes a stable long-term alteration in the epithelial cell phenotype [10], which in some cases promotes a faster rate cell cycling, increasing the number of dead cells [11]. The most acceptable hypothesis, however, is drug hypersensitivity, where the recall is caused by an idiosyncratic drug reaction activating inflammatory pathways that are not immune-dependent $[7,12]$.

In this report, we describe a case of RRD in a patient treated for breast cancer. The woman was operated for intraductal carcinoma one year before the onset of RRD and received prophylactic treatment with radiotherapy and tamoxifen. The RRD event reported here was triggered by oral acyclovir, an antiviral drug with viral replication inhibiting the activity of herpes simplex virus type 1 (HSV-1), 2 (HSV-2) and varicella-zoster virus (VZV) [3]. The drug has, as common adverse events, malaise, nausea, diarrhea, headache and, in rarer cases, immune thrombocytopenia [3]. However, the relationship with RRD has not yet been described.

\section{Case presentation}

A 54-year-old white woman sought medical attention in September 2015 in a city in the interior of the state of São Paulo with a complaint of spontaneous nipple bleeding associated with pain during physical exercise. She worked as a seamstress. The patient was married, had had four pregnancies, all cesareans, and no family history of breast cancer, but had a history of ovarian cyst surgical removal. She reported menopause at 42 years.

On physical examination, the breasts were symmetrical, with no nodules visualizations, architectural distortions or suspicion of calcifications. There was uniductal bloody papillary effusion on the left side, with the trigger point at 6 o'clock. Mammography and breast ultrasonography were performed, both coming with a BI-RADS 1 report. Despite imaging exams showing normality, the persistence of the suspicious serosanguinous secretion led the patient to be referred to the Regional Hospital's mastology outpatient clinic at Presidente Prudente, São Paulo state, with the indication of lumpecto$\mathrm{my} /$ resection of the main ducts in the left breast.

The surgical procedure resected a fragment of the breast's tissue for histopathological evaluation. The final diagnosis was intraductal carcinoma in situ of low grade, with a 3-mm single focus, of cribriform standard, with ductal ectasia, chronic mastopathy alterations, columnar cell alterations with foci of dystrophic calcification, and typical ductal hyperplasia with a $1-\mathrm{mm}$ single focus and free circumferential surgical margins. The immunohistochemistry evaluation of the same material assessed the expression of C-erbB-2 (Her-2 SP3), Ki-67 (Mab SP6), estrogen receptor (SP1) and progesterone Receptor (SP2). The immunohistochemistry profile result showed positivity for estrogen receptors in about $80 \%$ of neoplastic cells, progesterone receptors in about $30 \%$, high rate of cell proliferation measured by Ki-67 (over 14\%) and negativity for overexpression of epidermal growth Her-2 factor.

After hospital discharge, the patient was referred to radiotherapy, performed in 33 sessions, in the total dose of $5040 \mathrm{cGy}$ ( $180 \mathrm{cGy} /$ day). The patient also started the use of tamoxifen $20 \mathrm{mg} /$ day as hormonal therapy.

One year after the surgery, the patient visited a dermatologist complaining of sores on the left breast, local hardening and unchecked fever for about 15 days. She reported being diagnosed as having herpes zoster by a pharmacist in a drugstore near home and that she had started to use acyclovir (200 mg, every 6 hours during 14 days). During acyclovir use, breast pain started, and the 


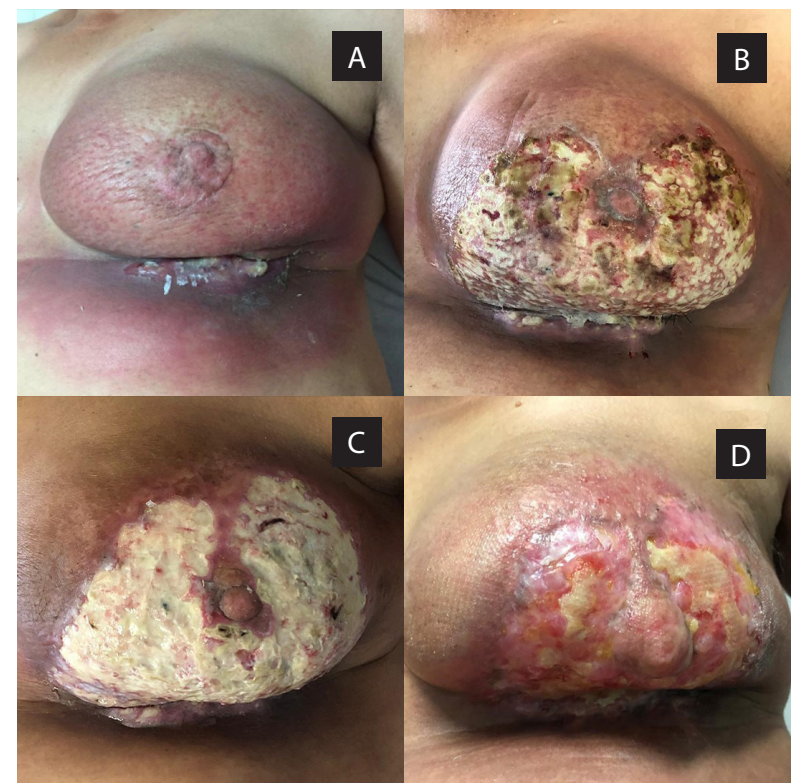

Figure 1. Clinical evolution of the patient with radiation recall dermatitis. A. Hyperemic breast with serosanguinolent secretion seen one year after breast cancer treatment.

B. Aspect of the breast after surgical debridement.

C. Supportive treatment with papain and SAF-gel.

D. Aspect of breast healing process between hyperbaric therapy sessions.

general clinical condition worsened. Because the patient self-medicated, there was no possibility to perform a microbiological analysis of pathological tissue to confirm herpes virus presence. The dermatologist noticed, on physical examination, erythema, skin hardening and infiltration throughout the left breast, and the presence of serosanguinolent discharge, without signs of herpes zoster or other skin problems. She was then referred back to the mastologist (Fig. 1A).

At the Regional Hospital, an investigation was conducted with chest and upper abdomen computed tomography, and biopsy of the dermal material from the left breast for anatomopathological examination. At the macroscopy exam, the first skin fragment taken for pathological examination was the size of $1.0 \mathrm{~cm} \times 0.6 \mathrm{~cm}$, with a finely grainy surface, brownish color and firm and elastic consistency; in the cuts, the surface was grayish-white. The second fragment was sized $0.6 \mathrm{~cm} \times 0.4 \mathrm{~cm}$, presented on the surface a $0.3 \mathrm{~cm}$ raised lesion, of vegetative aspect, gray-brown and firm and elastic consistency; in the cuts; the surface was grayish-brown.

The histopathological evaluation indicated acute erosive dermatitis, with vascular alterations suggestive of radiotherapy effects and absence of neoplasia

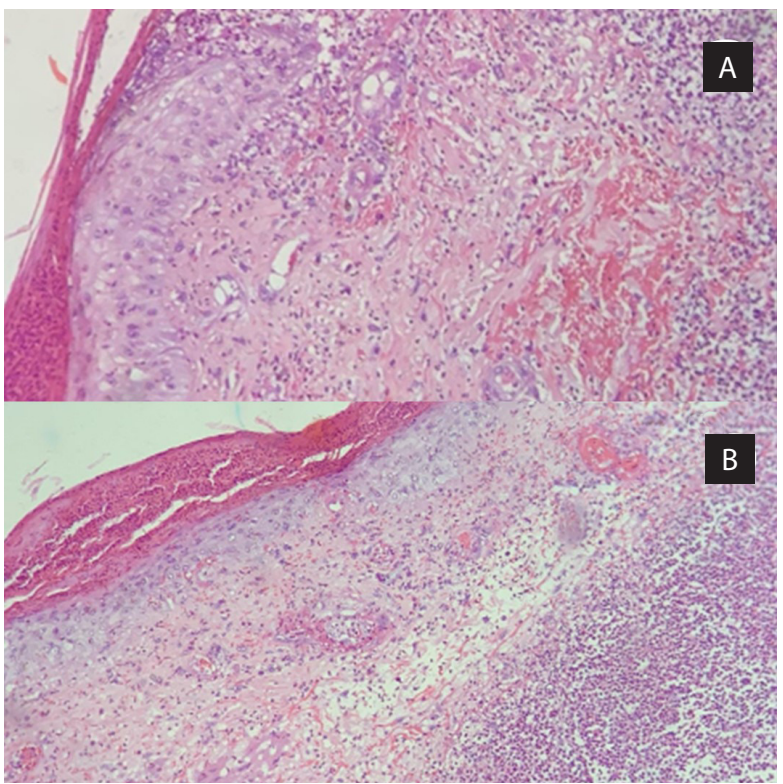

Figure 2. Histopathological evaluation of the patient with radiation recall dermatitis. A. Hematoxylin-eosin stain with $40 \times$ magnification showing intense neutrophilic exudate throughout the epidermis and dermis. B. Hematoxylin-eosin stain with $100 \times$ magnification showing ulcerated epidermis with foci of parakeratosis and acanthosis, liquefactive and atypical reactions of the basal layer. In the dermis, fibrosis and elastosis are noted, with intense fibrinoid exudate and vascular thickening with extravasation of red blood cells

(Fig. 2A and 2B). Imaging exams did not show signs of local or distant tumor recurrence. After complete anamnesis and complementary exams, the diagnostic hypothesis was radiation recall.

The patient was treated with repeated surgical debridements (Fig. 1B) associated with papain and SAF-gels (Fig. 1C) as recommended by the plastic surgery team. The patient was also referred for treatment with a hyperbaric chamber (Fig. 1D), and underwent 20 sessions. Breast reconstruction surgery with a graft has been scheduled.

\section{Discussion and Conclusion}

The patient in the RRD case reported here had a previous diagnosis of grade 2 carcinoma in situ with free margins and positive estrogen hormone receptor (ER-positive), treated with oncological surgery associated with radiotherapy and use of tamoxifen. According to Fischer et al., protocol B-17 [13], the addition of radiotherapy as an adjuvant treatment after surgery results in the reduction of local recurrence and, significantly, invasive carcinoma, playing a role as a preventive agent, since 
it destroys cancer cells and affects the precursors of these cells [13-15]. After 12 years of follow-up of patients submitted to the protocol B-17, the reduction in the annual incidence rate of all ipsilateral tumors, invasive or not, remained at $58 \%$ per year. The results found in the treatment of ductal carcinoma in situ by the European Organization for Research and Treatment of Cancer (EORTC), are similar to NSABP B17, in which a reduction in local recurrence rate was observed when the group submitted to the lumpectomy was treated with radiotherapy [15]. The use of tamoxifen, a selective estrogen receptor modulator [16], in patients with positive estrogen receptors provides a lower rate of ipsilateral recurrence, as well as a reduction in the occurrence of a new contralateral tumor and recurrence in the form of invasive carcinoma, according to the NSABP protocol B24 $[13,15,17]$. Therefore, both treatments were well justified for this patient at the time.

Only one report describes recall dermatitis related to the use of acyclovir, with some uncommon adverse skin reactions described with the use of acyclovir: vesicular dermatitis involving palms and soles, peripheral edema, erythema nodosum, rashes, hyperhidrosis, acne, lichenoid and skin rash, pruritus, urticaria, vasculitis and alopecia ${ }^{1}$. In that case, published in 2002, the rash appeared on the seventh day after oral acyclovir use, with small macules and erythematous papules involving the trunk and limbs symmetrically and bilaterally, along with the dermatomes previously affected by herpes varicella zoster [1]. However, the characteristics of active herpes varicella-zoster infection were not histopathologically verified by the authors, although the cutaneous eruption secondary to acyclovir was more intense in regions previously affected by the virus. Furthermore, the area of lesions had not been irradiated previously [1].

$\mathrm{RRD}$ is widely described in the literature, occurring in patients who underwent radiotherapy with symptoms precipitated after the use of specific drugs, such as actinomycin D, adriamycin, bleomycin, docetaxel, etoposide, 5-fluorouracil, gemcitabine, hydroxyurea, methotrexate, paclitaxel, simvastatin, tamoxifen, and antituberculosis medication, such as rifampicin, isoniazid and pyrazinamide, trimetrexate and vinblastine, and also other triggering agents, such as ultraviolet radiation [6,7]. The radiodermatitis presents in four degrees of toxicity: grade 1 (mild) with erythema, pruritus and dry flaking, grade 2 (mild-moderate) with pain, edema, urticaria or the appearance of vesicles, grade 3 (moderate) with wet desquamation and grade 4 (severe) with necrosis, ulcer or hemorrhage $[7,18]$.

The pathophysiology of RRD is unknown, but several hypotheses are described. One of the hypotheses is that changes in vascular permeability in the irradiated area would alter the drug's kinetics in that region, creating a hypersensitivity reaction, leading to acute inflammation [19]. Another accepted theory is related to radiation changes in localized cellular DNA, promoting the increased expression of inflammatory cytokines, such as interleukins 1 and 6 (IL-1, IL-6) and tumor necrosis factor-alpha (TNF-alpha), in the presence of the event-inducing drug [20].

The patient here described, after acyclovir use for the treatment of unproven herpes zoster infection, presented something different from a drug eruption: the onset of breast pain, dermal lesions and fever (not measured), progressing to grade 4 radiodermatitis, with serosanguinolent secretion accompanied by erythema, hardening and infiltration in the skin of the left breast, in areas previously irradiated. There was no sign of herpes zoster and no involvement of the breast tissue. Once the probable causative agent (acyclovir) was removed, and daily dressings and surgical debridement were performed, the patient's condition improved without complications. Imaging exams excluded the possibility of locoregional or distant tumor recurrence. The anatomopathological examination indicated acute erosive dermatitis with vascular alterations suggestive of radiotherapy effect and absence of neoplasia in the material, which suggested RRD due to the use of acyclovir as a diagnosis.

For the treatment of ductal carcinoma in situ, whenever possible, the conservative treatment is the choice, followed by radiation therapy and endocrine therapy to reduce the risk of local, contralateral recurrence and invasive carcinoma [13]. However, in some cases, total mastectomy is indicated, and immediate breast reconstruction should be considered [13]. To achieve a therapeutic success and prevent recurrence, clinical and imaging evaluation should be performed periodically, with patient informed about the risks.

The manifestation of RRD is described in different degrees of tissue involvement induced by 
different drug classes. However, it has not been described before in association with the use of acyclovir specifically. There are no precise descriptions of the pharmacological agents or classes of drugs that can induce RRD. Hence, for optimal diagnosis and management of patients with RRD, it is necessary to consider all the possible adverse effects of medications used after radiation, even months after radiotherapy. Also, it is advisable to use the lowest effective radiotherapy dose for the disease. This case report describes acyclovir as a possible trigger of radiation recall dermatitis, a rare condition that could have been mistaken for an eruption with other causes. In the present case, the dermatitis reaction was confined to the previously irradiated area of the skin, which suggested radiation recall dermatitis.

\section{Conflict of interests}

The authors declare that they have no competing interests.

\section{Funding}

None to declare.

\section{Authors' contributions}

M.T.Z. and R.M.P. wrote this report. R.S. and L.F.L. revised the manuscript. T.V.A.M. provided the images used in the report. M.T.Z., L.F.L., R.M.P. and R.S. saw the patient in hospital and contributed the case history notes used in this report. R.S. performed the clinical treatment and the lumpectomy. All authors read and approved the final manuscript.

\section{Acknowledgements}

We acknowledge our patient for providing informed consent for this case report and for her willingness to have her clinical experience published, Tatiana Veri de Arruda Mattos for the work in the Pathology Department in offering the original images and data related to this article and Rafael Sá for the clinical and surgical management of the patient.

\section{References}

1. Carrasco L, Pastor MA, Izquierdo MJ, et al. Drug eruption secondary to aciclovir with recall phenomenon in a dermatome previously affected by herpes zoster. Clin Exp Dermatol. 2002; 27(2): 132-134, doi: 10.1046/j.13652230.2002.00990.x, indexed in Pubmed: 11952706.
2. Azria $D$, Magné $N$, Zouhair $A$, et al. Radiation recall: a well recognized but neglected phenomenon. Cancer Treat Rev. 2005; 31(7):555-570, doi: 10.1016/j.ctrv.2005.07.008, indexed in Pubmed: 16168567.

3. Hong $X$, Wang $X$, Wang Z. A rare case report of acyclovirinduced immune thrombocytopenia with tongue hematomas as the first sign, and a literature review. BMC Pharmacol Toxicol. 2017; 18(1): 12, doi: 10.1186/s40360017-0120-2, indexed in Pubmed: 28264696.

4. Simon A, Robb K. Cancer: Breast. Cambridge Handbook of Psychology, Health and Medicine. 2nd ed. Cambridge University Press, Cambridge 2014: 577-580.

5. Sharma GN, Dave R, Sanadya J, et al. Various types and management of breast cancer: an overview. J Adv Pharm Technol Res. 2010; 1(2): 109-126, indexed in Pubmed: 22247839.

6. Boström A, Sjölin-Forsberg G, Wilking N, et al. Radiation recall $\triangle$ another call with tamoxifen. Acta Oncol. 1999; 38(7): 955-959, doi: 10.1080/028418699432653, indexed in Pubmed: 10606426.

7. Camidge R, Price A. Characterizing the phenomenon of radiation recall dermatitis. Radiother Oncol. 2001; 59(3): 237-245, doi: 10.1016/s0167-8140(01)00328-0, indexed in Pubmed: 11369064.

8. Hellman S, Botnick L. Stem cell depletion: An explanation of the late effects of cytotoxins. Int J Radiat Oncol Biol Phys. 1977; 2(1-2): 181-184, doi: 10.1016/03603016(77)90028-1, indexed in Pubmed: 849898.

9. Seymour CB, Mothersill C, Alper T. High yields of lethal mutations in somatic mammalian cells that survive ionizing radiation. Int J Radiat Biol Relat Stud Phys Chem Med. 1986; 50(1): 167-179, doi: 10.1080/09553008614550541, indexed in Pubmed: 3487520.

10. Wright EG. Radiation-induced genomic instability in haemopoietic cells. Int J Radiat Biol. 1998; 74(6): 681-687, doi: 10.1080/095530098140943, indexed in Pubmed: 9881712.

11. Abadir R, Liebmann J. Radiation reaction recall following simvastatin therapy: a new observation. Clin Oncol (R Coll Radiol). 1995; 7(5): 325-326, doi: 10.1016/s09366555(05)80545-x, indexed in Pubmed: 8580062.

12. Wintroub B, Stern R. Cutaneous drug reactions: Pathogenesis and clinical classification. J Am Acad Dermatol. 1985; 13(2): 167-179, doi: 10.1016/s0190-9622(85)70156-9, indexed in Pubmed: 2931455.

13. Fisher B, Costantino J, Redmond C, et al. Lumpectomy compared with lumpectomy and radiation therapy for the treatment of intraductal breast cancer. N Engl J Med. 1993; 328(22): 1581-1586, doi: 10.1056/ NEJM199306033282201, indexed in Pubmed: 8292119.

14. Fisher B, Dignam J, Wolmark N, et al. Lumpectomy and radiation therapy for the treatment of intraductal breast cancer: findings from National Surgical Adjuvant Breast and Bowel Project B-17. J Clin Oncol. 1998; 16(2): 441-452, doi: 10.1200/JCO.1998.16.2.441, indexed in Pubmed: 9469327.

15. Fisher B, Land S, Mamounas E, et al. Prevention of invasive breast cancer in women with ductal carcinoma in situ: an update of the National Surgical Adjuvant Breast and Bowel Project experience. Semin Oncol. 2001; 28(4): 400-418, doi: 10.1016/s0093-7754(01)90133-2, indexed in Pubmed: 11498833. 
16. Patterson J, Furr B, Wakeling A, et al. The biology and physiology of 'Nolvadex' (tamoxifen) in the treatment of breast cancer. Br Cancer Res Treat. 1982; 2(4): 363-374, doi: 10.1007/bf01805878.

17. Rhee J, Kim GE, Lee $\mathrm{CH}$, et al. Radiation recall dermatitis induced by tamoxifen during adjuvant breast cancer treatment. Radiat Oncol J. 2014; 32(4): 262-265, doi: 10.3857/ roj.2014.32.4.262, indexed in Pubmed: 25568855.

18. Mehta K, Kaubisch A, Tang J, et al. Radiation Recall Dermatitis in Patients Treated with Sorafenib. Case Rep Oncol
Med. 2018; 2018: 2171062, doi: 10.1155/2018/2171062, indexed in Pubmed: 29670787.

19. Phillips T, Fu K. Quantification of combined radiation therapy and chemotherapy effects on critical normal tissues. Cancer. 1976; 37(S2): 1186-1200, doi: 10.1002/1097-0142(197602)37:2+<1186::aidcncr2820370830>3.0.co;2-v, indexed in Pubmed: 766958.

20. Oanță A, Irimie M. Radiation Recall Dermatitis induced by Tamoxifen. Med Sci. 2012; 5(54): 113-116. 